\title{
Interaction effects in successive visual displays: An extension of the Eriksen-Collins paradigm
}

\author{
IRWIN POLLACK* \\ Wental Health Research Institute, University of Michigan, Ann Arbor, Michigan 48104
}

\begin{abstract}
Th. paradigm of Eriksen and Collins, in which one-half of the elements of a display is assigned randomly to each of two successively presented fields, was applied to the visual discrimination of one- and two-dimensional Markov constraints. In contrast with the two-flash masking paradigm, performance in the Eriksen-Collins paradigm improves as the interval between the successively presented fields is shortened. Constraint thresholds may increase with interfield intervals as short as $20 \mathrm{msec}$. In contrast with the results of most visual information-processing tasks, increasing the duration of each field may result in sharply eleveted thresholds. The elevation of thresholds can be reduced through repeated presentation. Sharp asymmetries are also obtained with differential brightness of the two fields.
\end{abstract}

The two-field paradigm has become an extremely widespread tool in the study of visual perception. Depending upon the task and the visual parameters, a variety of interactive effects have been described. A number of excellent review sources are available, e.g., Kahneman (1969).

When negative or interference effects obtain, concepts such as masking, metacontrast, erasure, noise, luminance summation, and overprinting have been defined for the two-flash paradigm, and sequential blanking and sequential displacement have been defined for the multiflash paradigm. When positive or noninterference effects obtain, concepts such as luminance summation. integration, and additivity have been defined.

A complete theory of the two-field paradigm will also include task variables. The informational contribution of each of the separate display fields to the definition of the overall task may be expected to be crucial. If the separate fields each contribute information to the task under study, an integration or pooling of the separate fields is indicated. Under these circumstances. performance would be expected to improve as the interfield interval is decreased. However. if only one field contributes to the task under study-as in the typical definition of a "signal" field and a "masking" field-isolation of the separate fields is indicated. Under these circumstances. performance would be expected to improve as the interfield interval is increased.

An important approach to the informational integration of two display fields is the "hidden figure" paradigm of Eriksen and Collins (1967. 1968). Nonsense syllables are constructed out of dots and are embedded in a background of random dots. With special care in

\footnotetext{
*The research was supported in part by Grant GB $14036 \mathrm{X}$ from the National Science Foundation. The writer is indebted to Nancy Mandell for supervising the experimental tests, to Eva Williams-Malcolm for supervising the control tests. to Louis Wojnaroski for the computer program. and to Robert Shea for processing the experimental results.
}

parcelling out the dots into two separate display fields, the following results were obtained in the Eriksen-Collins study: when selected dots were assigned to the separate visual fields, the resulting display was nearly illegible; when the two fields were directly superimposed, the display was highly legible. Identification of the nonsense syllable fell sharply as the interfield interval, or IFI, was extended beyond $25 \mathrm{msec}$. Had the materials been divided, as in masking studies, with the nonsense syllable within Field 1 and with the random dots within Field 2, identification presumably would have improved as the interfield interval was extended.

The present study extends the Eriksen-Collins paradigm to the detection of one- and two-dimensional Markov constraints within visual displays. Such displays can be quantitatively described in terms of local probabalistic contingencies. can be constructed by computational algorithms without careful editing of the elements into separate fields, and may provide a more general method for evaluating the interaction of two visual informational fields than does the interleaving of nonsense syllables of Eriksen and Collins.

\section{METHOD}

The method of generating one-dimensional and two-dimensional Markov constraints within visual displays has been described previously (literature citations in Pollack, 1972). The methods will not be reviewed in detail. It is sufficient to note that one-dimensional strings of dots and nondots are constructed by varying the conditional repetition probability, CRP. The CRP is the probability that a dot will follow a dot or that a nondot will follow a nondot, and will be abbreviated as PiA A).

One-dimensional constraints were plotted within 32 by 32 matrices with an interrow and intercolumn spacing of $1.17 \mathrm{~mm}$. The direction of the displayed constraint was vertical. The initial row of the matrix was a random unconstrained sequence of dots and nondots. The dots were randomly assigned to two part fields. Which were presented successively in time. When each part field contains half of the dots. we shall refer to it as a half field. 


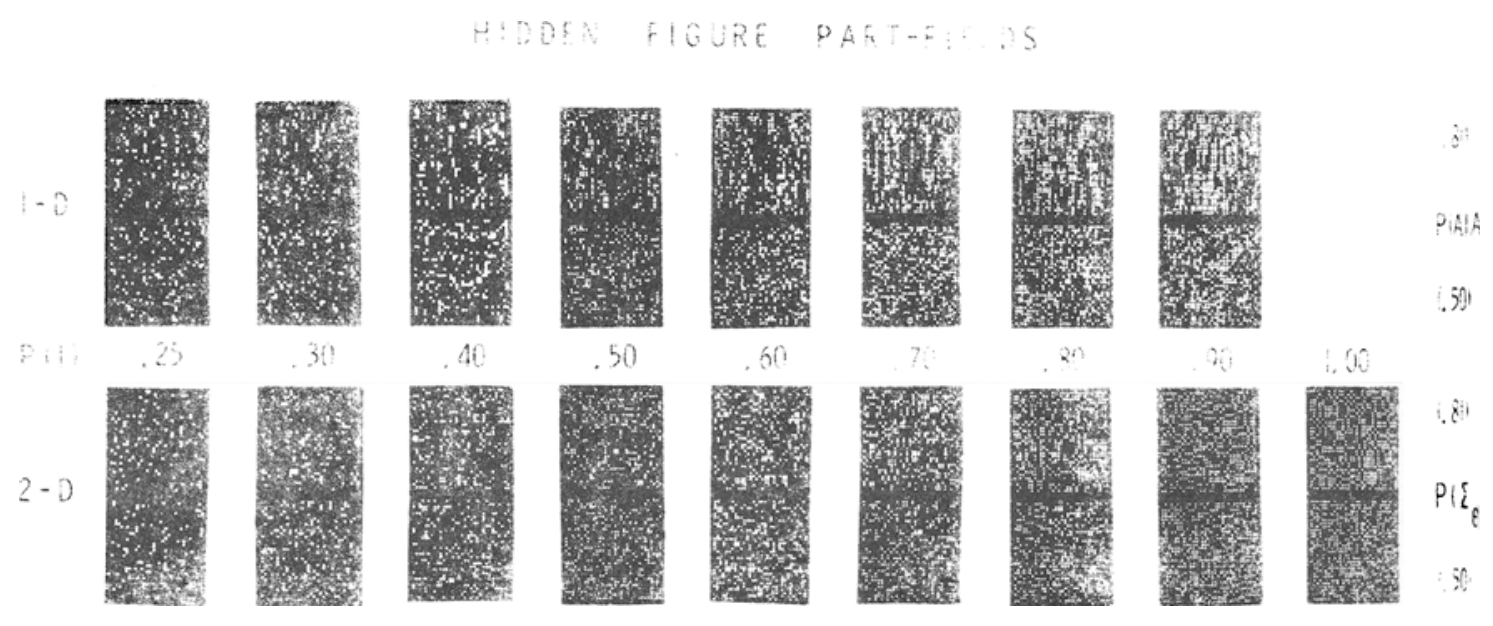

Fig. 1. Photographs of one-dimensional (top row) and two-dimensional (lower row) Markov spatial constraints as a function of the proportion of the total dots painted within the first part field, $P(1)$. Within each row, the top section represents a constrained selection with $P(A \mid A)$ or $P\left(S_{e}\right)=.8135$; the bottom section represents an unconstrained selection with $P(A \mid A)$ or $P\left(S_{e}\right)=.5000$. In a given test, four matrices were displayed within the same field: three at one $P(A \mid A)$ or $P\left(S_{e}\right)$ level and one at a different level. The task of the $O$ was to select the position of the odd display. Note that the linear one-dimensional constraints can be discriminated from the reference level at a lower proportion of the total dots than can the two-dimensional constraints. (Differences in size and density were not present in the initial displays.)

The top line of Fig. 1 illustrates the first of two part fields of a hidden-figure display for one-dimensional Markov constraints. Within each box, two displays are pictured: the top one at $P(A \mid A)=0.8135$ and the bottom one at $P(A \mid A)=0.5000$. The entry is the proportion of the total dots assigned to the first part field, $P(1)$. The other part field, $P(2)$ (not shown), employed the remainder of the dots, or $P(2)=[1.0-P(1)]$.

Two-dimensional constraints were generated by varying the probability of a fixed parity sum over overlapping 2 by 2 cells within the matrix. An even parity sum leads to 0,2 , or 4 dots within the 2 by 2 cell; an odd parity sum leads to 1 or 3 dots. Given the previous specification of 3 of the 4 elements in a 2 by 2 array, the fourth element is specified by the parity sum. For example, the top row and left column of the 32 by 32 matrix were unconstrained random sequences. Three of four elements in the upper left corner are specified. The fourth can be specified by the parity sum. The probability of an even parity sum is abbreviated as $\mathbf{P}\left(\mathrm{S}_{\mathrm{e}}\right)$.

The bottom line illustrates the first of two part fields of a hidden-figure display for two-dimensional Markov constraints. As in the top line, two displays are pictured within each box, the top one at $\mathrm{P}\left(\mathrm{S}_{\mathrm{e}}\right)=0.8135$ and the bottom one at $\mathrm{P}\left(\mathrm{S}_{\mathrm{e}}\right)=$ 0.5000 . The entry is the proportion of the total dots assigned to the first part field. The other part field (not shown) employs the remaining proportion of dots, or $[1.0-\mathrm{P}(1)]$.

The displays were painted upon a high-speed display scope (Tektronix 602 with a fast P15 phosphor) by a PDP-9 computer (Digital Equipment Corporation). Upon each observation, four matrices were painted in a 2 by 2 array upon an $8 \times 8 \mathrm{~cm}$ display area. Three of the matrices employed a reference set of Markov constraints, $\mathbf{P}(\mathbf{A} \mid \mathbf{A})_{\mathbf{R}}$ in one-dimensional tests or $\mathbf{P}\left(\mathrm{S}_{\mathbf{e}}\right)_{\mathbf{R}}$ in two-dimensional tests. The fourth employed a different set, $P(A \mid A) v$ or $P\left(S_{e}\right) v$. The task of the $O$ was to identify the "odd" matrix by pressing one of four buttons in a matching 2 by 2 array. An adaptive stimulus programming procedure (Taylor \& Creelman, 1967) varied $P(A \mid A)_{V}$ or $P\left(S_{e}\right)_{V}$ in order to converge upon $50 \%$ correct response in the four-alternative forced-choice (4AFC) test. Under conditions of difficult discrimination, the adaptive procedure might seek a constraint level beyond 1.0. One observation was provided at the extreme condition; if incorrect, the trial was terminated. With $<10 \%$ terminated trials, averages are geometric means over nonterminated trials. With $\geqslant 10 \%$ terminated trials, averages are medians including the terminated trials. Conditions with $10 \%-20 \%$ of such trials are shown as half-filled points; conditions with $\geqslant 20 \%$ of such trials are shown as filled points. The degree of shading, then, represents a crude measure of discriminability.

Displays were painted at $9 \mu \mathrm{sec}$ per point by a high-speed plotting display routine (Wojnaroski et al, 1971). Four 32 by 32 display matrices, with a dot probability of 0.5 and with half of the points in each of the two display fields, required $(4)(1,024)(0.5)(0.5) 9 \mathrm{sec}$ or $9.2 \mathrm{msec}$ to plot. Since points were plotted in a fixed geographic sequence, successive plotting of the same points, or of neighboring points, were spaced by the time required to paint the entire display.

In the most general case, Proportion $\mathrm{P}(1)$ of the total number of dots was assigned to the first display and was plotted $t_{1}$ times. After a wait, or IFI, the dots assigned to the second display, $P(2)$ $=1.0-P(1)$, were plotted $t_{2}$ times. After another IFI, the entire cycle of first field, IFI, second field, IFI was repeated $R$ successive times.

The paid Os had extensive experience in psychophysical testing (up to six semesters, $5-10 \mathrm{~h} /$ week) or had developed their experience during the extended testing program. Each point represents two thresholds by each of $15 \mathrm{Os}$. In all, 594 experimental conditions were examined, resulting in about $1.8 \mathrm{x}$ $10^{4}$ thresholds.

\section{RESULTS}

$$
\text { Single Part Fields, } P(2)=0
$$

One of the requirements of the Eriksen-Collins hidden-figure paradigm is that the separate part fields are more poorly discriminated than is their combination. Figures 2 and 3 examine the discriminability of one- and two-dimensional Markov constraints for a single part field as a function of the proportion of the total number of dots painted in that part field (as in Fig. 1). The complementary part field was suppressed. The parameter on the curves is the exposure duration.

Figure 2 shows that one-dimensional constraint 
thresholds are sensitive to the proportion of the total dots actually displayed and are relatively independent of display duration. For the special cases of $P(1)=0.50$ and 1.0 , however, there is only a relatively narrow range of constraint thresholds.

By contrast, Fig. 3 shows that two-dimensional constraint thresholds are sensitive to exposure duration, improving with longer durations of the part field. More importantly for our present purposes, thresholds at $\mathrm{P}(1)$ $=0.50$ are either extremely large or are attainable only with a large proportion of terminated trials. [The multiplicity of points at $\mathrm{P}(1)=0.50$ represents independent replications.]

Since there is a potentially wider range of thresholds to be obtained within the hidden-figure paradigm with two-dimensional constraints than with one-dimensional constraints, we shall report only the results with 2-D constraints. The major findings were replicated with 1-D constraints, although the range of thresholds was necessarily abbreviated.

\section{Two-Dimensional Constraints}

Figure 4 considers the detection of two-dimensional constraints within the hidden-figure paradigm as a function of the number of replications of the first and second fields (parameter) and as a function of the display duration (abscissa) per replication. No additional delay, or IFI, was introduced between the displays. With a single replication, $\mathrm{R}=1$, thresholds increase with longer display durations. Thresholds also decrease with successive repetitions of the sequence: first half field, second half field, first half field, ... with minimum thresholds obtained at intermediate display durations in the region of two to six 9-msec frames.

Is best integration of the half fields obtained with

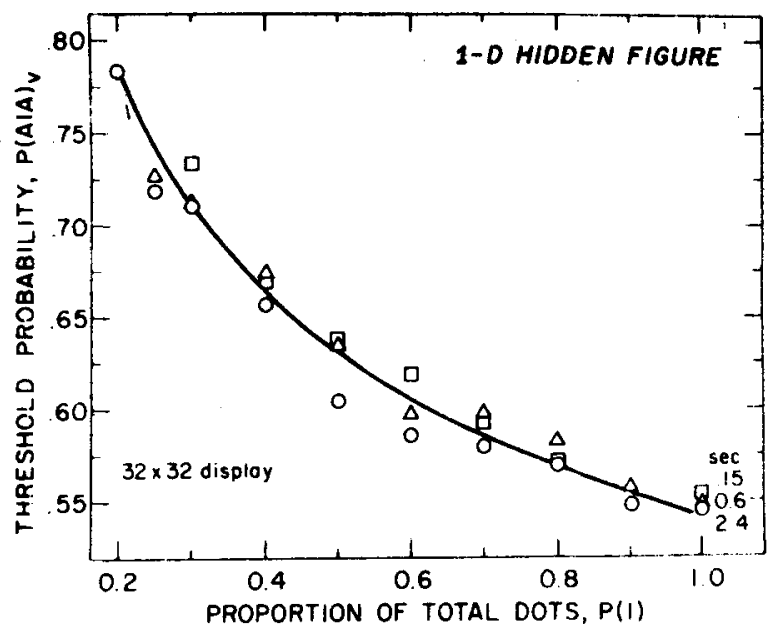

Fig. 2. Thresholds for one-dimensional Markov constraints as a function of the proportion of the total dots presented within a single part field (see Fig. 1). The second part field was suppressed. The parameter is the duration of the display.

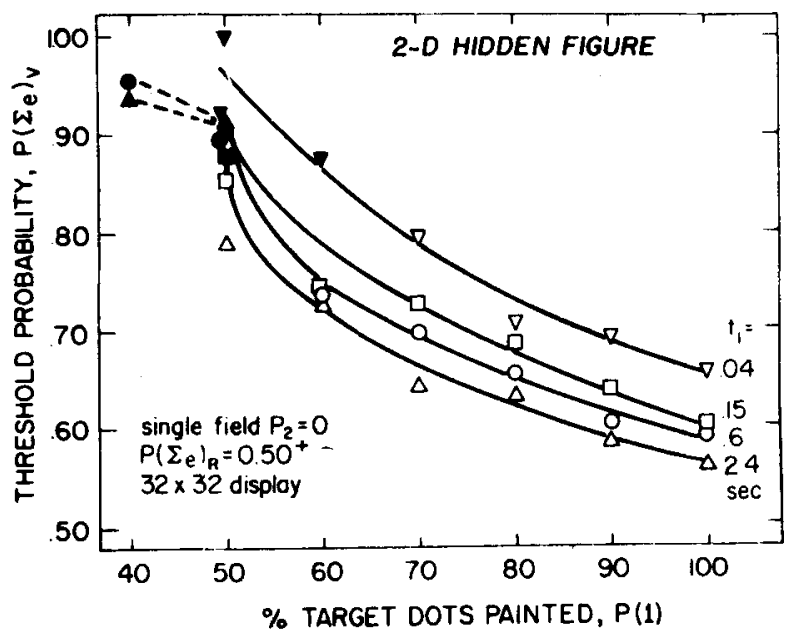

Fig. 3. Thresholds for one-dimensional constraints as a function of the proportion of the total dots presented within a single part field (see Fig. 1). The second part field was suppressed. The parameter is display duration. The shading of the points represents a crude measure of discrimination difficulty.

equal-duration half fields? Figure 5 examines the $t_{1}: t_{2}$ interaction for the discrimination of 2-D constraints without additional delays. Lowest thresholds are obtained at $t_{1}=t_{2}$. (Not shown are thresholds with 2:1 and $1: 2$ ratios, which fall between the $3: 1$ and $1: 1$ or

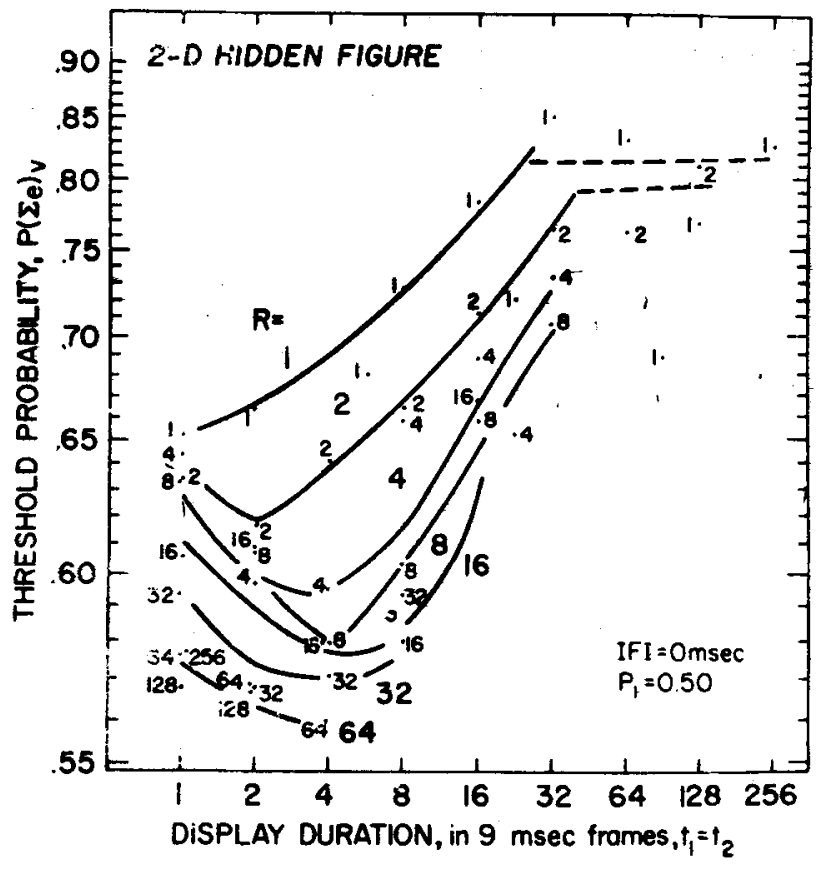

Fig. 4. Thresholds for two-dimensional constraints as a joint function of the duration of each half field and of the number of repetitions of the sequence of the two half fields, $R$. The ordinate is based upon a logarithmic scaling only for the purpose of spreading out the lower points. In Figs. 4-8, $P(1)=P(2)=0.5$ and $P\left(S_{e}\right)_{R}=0.5$. 


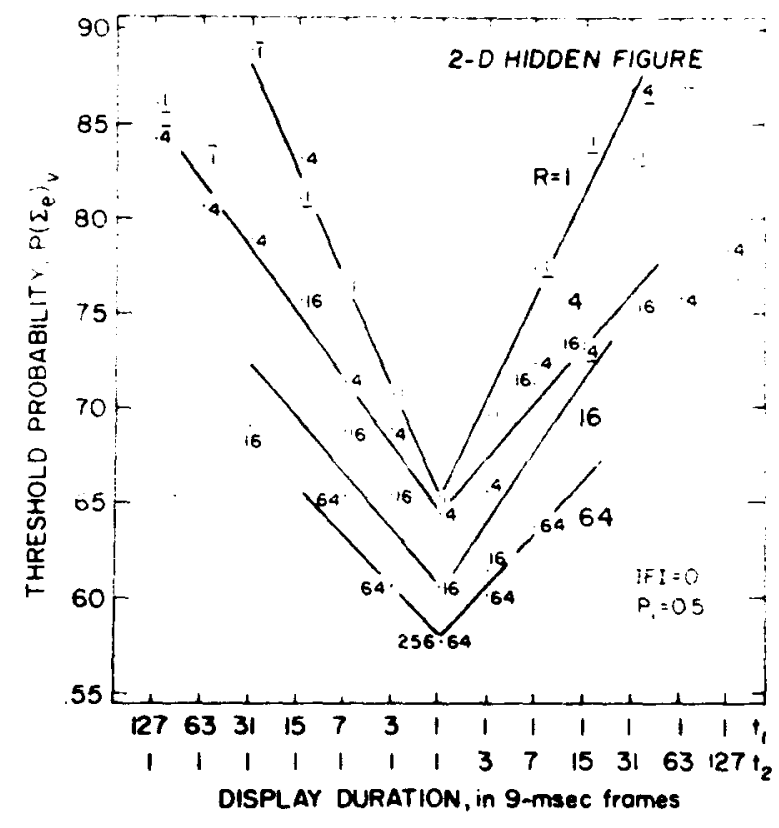

Fig. 5. Thresholds for two-dimensional constraints as a function of the duration of the two half fields. The parameter is the number of repetitions of the sequence of the half fields.

1:I and 1:3 ratios.) Successive replications-the parameter on the curves-again brings about a progressive lowering of thresholds.

The increase in thresholds with longer duration half fields noted in Fig. 5 is not restricted to part fields of equal duration. The sharp changes in thresholds noted in Fig. 5 with unequal presentations may be suspect because one of the half fields was plotted for a single 9-msec frame. Are the sharp asymmetries preserved under a simple multiplicative transform? For example, under multiplicative transform $M=1,4$, and 16 , the $t_{1}: t_{2}$ ratio of $3: 1$ becomes $t_{1}=3, t_{2}=1 ; t_{1}=12, t_{2}=4$; and $t_{1}=48, t_{2}=16$. When this is done, not only are the asymmetries of Fig. 5 preserved, but thresholds increase at larger $M$ values. The threshold increment, relative to $\mathrm{M}=1$, averaged about .04 for $\mathrm{M}=4$, about .12 for $\mathrm{M}=$ 16 , and about .19 for $M=64$. The sharp changes in threshold with unequal presentation in Fig. 5, therefore, are not limited to exceedingly brief presentations.

\section{Additional Waiting Time, IFI $>0$}

To this point, we have considered only successively presented displays without additional delays between the two part fields within the hidden-figure paradigm. Figure 6 samples the effect of additional waiting time for matrices of different number of elements. The approximate painting time for each part field was $9 \mathrm{msec}$ ( 32 by 32 matrix), $4.5 \mathrm{msec}$ ( 23 by 22 ), $2.25 \mathrm{msec}(16$ by 16), $1.12 \mathrm{msec}$ ( 12 by 11 ), and $0.56 \mathrm{msec}$ ( 8 by 8 matrix). With a zero IFI, neighboring points within any local region are separated by the painting time of each part field. Successive curves are displaced upward to represent successively smaller displays.

Two-dimensional constraint thresholds are sensitive to IFI. the interval between two successive part fields. Thresholds increase beyond IFIs of $16 \mathrm{msec}$ for matrices of all sizes. A substantial fraction of trials must be terminated for IFIs as low as $30 \mathrm{msec}$ for the 12 by 11 matrix ranging to $115 \mathrm{msec}$ for the 32 by 32 matrix. In confirmation of the results of Eriksen and Collins, the fall-off in performance with IFI is extremely rapid within this paradigm.

The deterioration in thresholds with IFI shown in Fig. 6 was extended to 32 by 32 displays with $t_{1}=t_{2}=$ 1 to 64 in octave steps (or to display durations of 9 to $576 \mathrm{msec}$ ). Except for the threshold increments associated with the longer display's, threshold changes with IFI were maintained for $t_{1}=t_{2}=1,2,4$, and 8 . At longer display durations, $t_{2}=t_{2}=16$ to 64 , thresholds were less dependent upon the IFI. In addition, the effect of successive replications of the entire sequence-first half field, wait, second half field, wait-produced a lowering of thresholds of the order of that shown in Figs. 4 and 5.

Finally, we sought to determine whether the strong effects of unequal duration, shown in Fig. 5, are observed when an IFI is introduced. The three sections of Fig. 7 represent IFIs of 4,16 , and $64 \mathrm{msec}$. The parameter is the number of paintings of the first part field (in 9-msec steps); the abscissa is the number of paintings of the second part field. Within each panel, thresholds with $t_{1}=1$ and 64 paintings are connected by

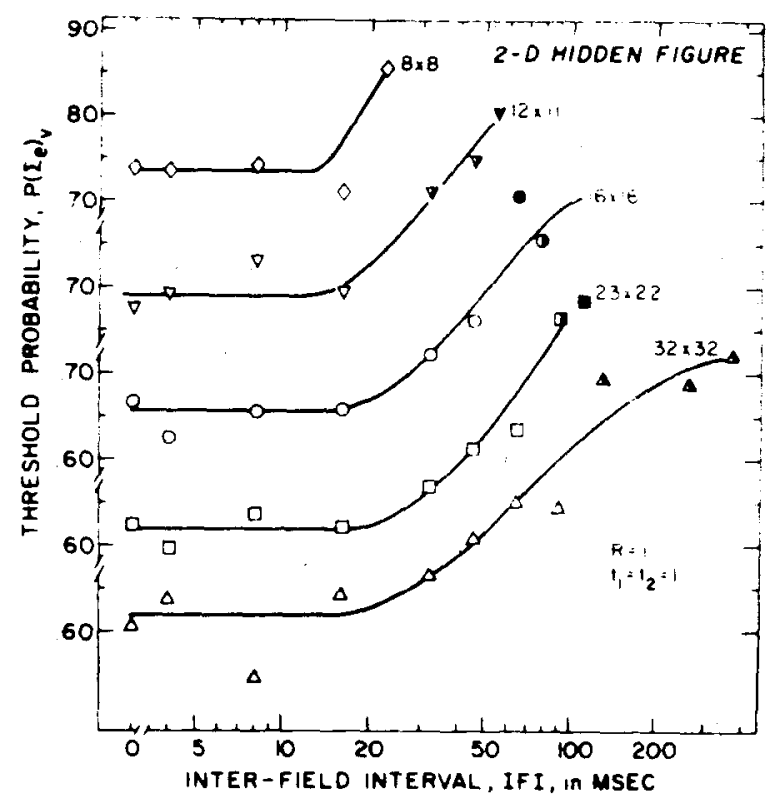

Fig. 6. Thresholds for two-dimensional constraints as a function of the IFI between successive half fields for matrices of varying size (parameter). Successive curves are displaced upward to provide nonoverlapping displays. 


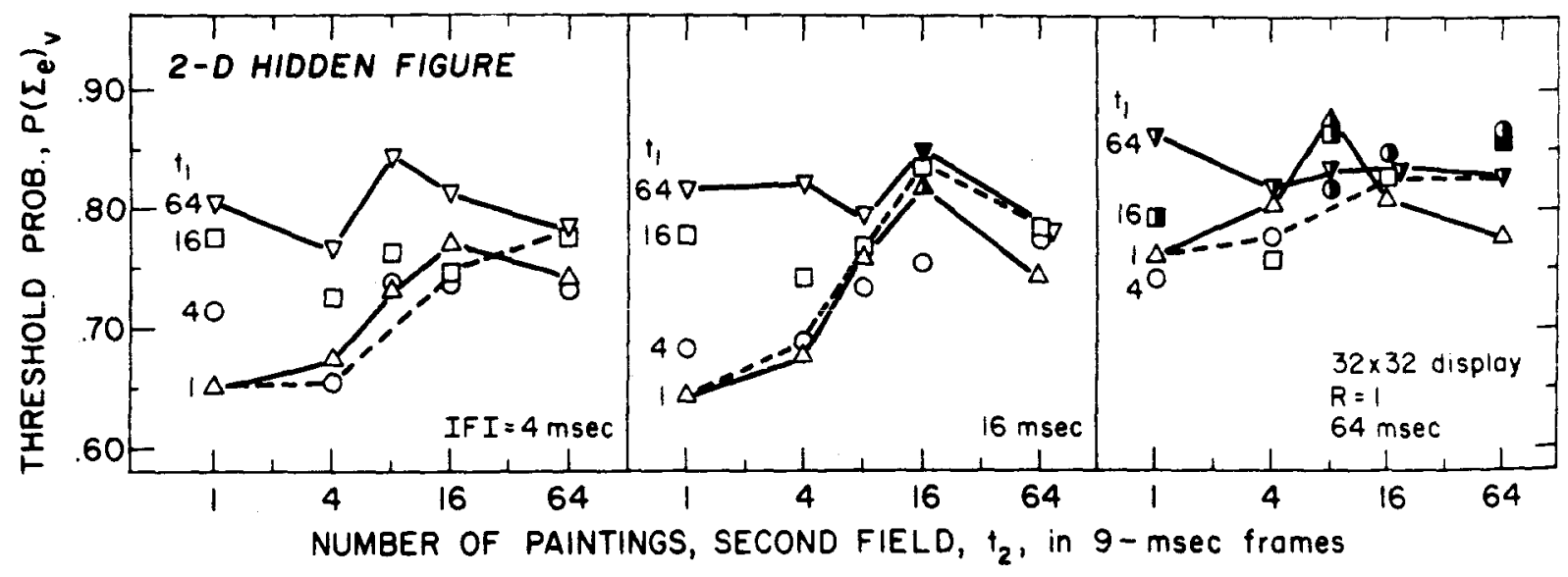

Fig. 7. Thresholds for two-dimensional constraints as a function of the number of paintings-in 9-msec frames-for the first part field (parameter) and second part field (abscissa). The three sections represent three IFIs. The solid lines connect points with $t_{1}=64$ and with $t_{1}=1$. The dashed lines connect points with $t_{1}=t_{2}$.

solid lines; the dashed line connects points where $t_{1}=$ $t_{2}$. The main result is that thresholds increase either as $t_{1}$ or $t_{2}$ increases.

It is not clear whether the interactive effects necessarily become attenuated with longer IFIs or whether the apparent attenuation results from a more restricted range of thresholds. For the shortest IFIs, there is a suggestion that near-minimum thresholds are obtained with $t_{1}=t_{2}$, as noted earlier in Fig. 5 .

\section{DISCUSSION}

In the absence of extraneous visual fields ("noise"), the viewing conditions favored the persistence of previously presented materials. Such persistence might have been expected to bridge the temporal gap between the two part fields in the interleaved-element procedure. With the reasonable assumption that persistence is proportional to the duration of the first field, better performance was anticipated with the longer display fields. These reasonable expectations were disconfirmed. These results are especially interesting since constraint thresholds decrease unambiguously with longer durations of a single part field, especially with the 2-D constraints.

In the previous tests, display brightness was directly confounded with display duration: the longer the display, the greater the number of paintings of the part field. Additional control tests were run with new Os in which blank fields were introduced to partially unconfound the number of paintings. $p$, with display duration, d. For example, the schematic insert in the lower left corner of Fig. 8 represents a part field, painted three times. with a single blank field interposed between each painting. For this example, $p=3, b=1, d=5$ : and. in general. $d=p+(p-1) b$. Without blank fields in the earlier tests, $\mathrm{t}=\mathrm{d}=\mathrm{p}$. As in Fig. 5, conditions were selected with $p_{2}=1$. which was combined with a range of $p_{1}$ levels. and with $p_{1}=1$, which was combined with a range of $p_{2}$ levels. A new group of Os contributed a pool of 40 thresholds over each of 41 experimental conditions. The circles and straight lines in Fig. 8 represent the baseline condition in which $b=0$, as in Fig. 5, where $t=d=p$.

Figure 8 tests two hypotheses which are labeled a "brightness" hypothesis and a "duration" hypothesis. The brightness hypothesis assumes that performance is related to the number of paintings of each half field, irrespective of the number of blank intervals. (The relation between apparent brightness and the number of paintings is not entirely direct, because the apparent brightness suffers with a large number of blank intervals.) The left section of Fig. 8-in which the abscissa displays the number of paintings of the first half field, $p_{1}$, or of the second half field. $p_{2}$-tests the brightness hypothesis. If thresholds were determined by $p_{1}$ and $p_{2}$, the points of the left section would congregate about the circles and straight lines.

The duration hypothesis assumes that performance is related to the total elapsed duration of each half field, irrespective of the number of blank intervals. The right section of Fig. 8-in which the abscissa displays the duration of the first half field, $d_{1}$, and the duration of the second half field, $d_{2}-$ tests the duration hypothesis. If thresholds were determined by $d_{1}$ and $d_{2}$. the points of the right section would congregate about the circles and straight lines.

The finding of higher thresholds with greater asymmetry, noted in Fig. 5, is clearly replicated. Moreover, thresholds are more closely associated with the number of display paintings in the left section (the brightness hypothesis) than with the total display duration in the right section (the duration hypothesis).

An appeal to "psychoneurology" might suggest that each local region that is stimulated establishes an "excitatory field" as well as an "inhibitory field" in that local region. perhaps as described by Békésı (1958) under the concept of "funneling." Additional 


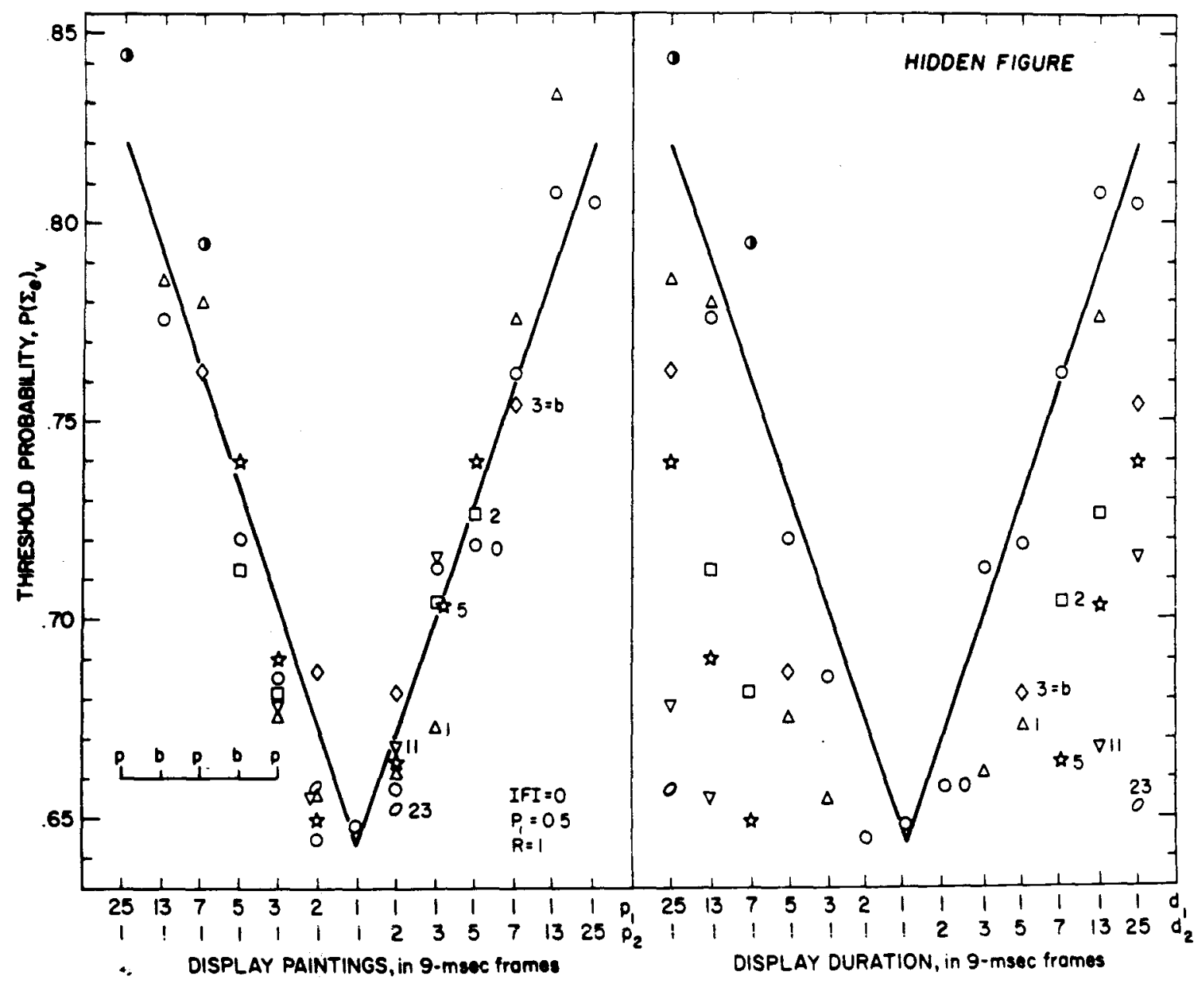

Fig. 8. Thresholds for two-dimensional constraints as a function of (left section) the number of paintings of the two half fields, $p$, and of (right section) the total display duration of the two half fields, $d$. The parameter is the number of blank half fields introduced between successive paintings. The insert in the lower left corner represents: $p=3, b=1, d=5$.

assumptions are required with respect to the time courses of the excitatory and inhibitory fields. A plausible notion would be that the excitatory field is quickly established and gains only little with further exposure, whereas the inhibitory field is slowly established and gains appreciably with further exposure. While such hypothetical psychoneurology has an "explanatory" flavor for some of the results, we must be careful in our response to this apparently soothing siren song. The paradox of poorer performance with increased display duration in an information processing task seems important enough to require explanation in its own terms. I had originally felt that the solution of the paradox lay in a process which, once started, proceeds to termination. irrespective of display duration ( $E f_{\text {Ion, }}$ 1970). However, Fig. 8 suggests that the relevant variable is not display duration, but a variable (brightness?) related to the number of display paintings.

The results bear upon three hypotheses discussed by Eriksen and Collins (1968).
The sensory-trace hypothesis assumes lowest thresholds when the traces of the two fields are at equal brightness at their moment of integration. Since the first field has a longer time to fade relative to the second, this hypothesis would favor brighter first fields relative to brighter second fields. This prediction is disconfirmed.

The perceptual moment hypothesis assumes that incoming information cannot be integrated across successive moments. This hypothesis would favor short first display fields and would predict equivalent performance for first display fields greater than one moment in duration. The latter prediction is disconfirmed for a wide range of hypothetical moments.

The discontinuity hypothesis assumes that discontinuity detectors interrupt temporal integration across successive display fields. This notion is attractive because independent studies of auditory discontinuity or gap detection, for example (Pollack. 1967, 1970), show that gap thresholds increase as the first and second display fields are displaced in quality. as the separate 
display fields are made successively shorter and shorter. and as the separate fields become more and more asymmetrical. These findings roughly parallel those of the present study. Visual temporal gap thresholds (Uttal \& Hieronymus, 1970), however, may show nonmonotonic relations. Nevertheless, discontinuity or gap detection may be sufficiently related to information integration in the Eriksen-Collins paradigm to warrant the examination of both tasks within a common experimental framework.

\section{REFERENCES}

Békésy. G. von. Funneling in the nerrous sytem and its role in loudness and sensation intensity on the skin. Journal of the Acoustical Society of America, 1958, 30, 399-412.

Efron, R. Effect of stimulus duration on perceptual onset and offset latencies. Perception \& Psychophysics, 1970. 8. 231-234.

Eriksen, C. W., \& Collins, J. F. Some temporal characteristics of visual pattern perception. Journal of Experimental Psychology, 1967. 74, 476.484.

Eriksen. C. W., \& Collins. J. F. Sensory traces versus the psychological moment in the temporal organization of form. Journal of Experimental Psychology, 1968, 77. 376-382.

Kahneman, D. Method, findings and theory in studies of visual masking. Psychological Bulletin, 1968, 70, 404-425.

Pollack. I. Asynchrony: The perception of temporal gaps within periodic auditory julse patterns. Journal of the Acoustical Society of America: 1967, 42, 1335-1340.

Pollack, I. Proximity requirements for auditory gap detection. Perception \& Psychophysics. 1970, 7, 137-142.

Pollack, I. Visual discrimination thresholds for one- and two-dimensional Markov spatial constraints. Perception \& Psychophysics. 1972, 12. 161-167.

Taylor, M. M., \& Creelman, C. D. PEST: Efficient estimates on probability functions. Journal of the Acoustical Society of America, 1967, 41, 782-787.

Uttal, W. R., \& Hieronymus. R. Spatio-temporal effects in visual gap detection. Perception \& Psychophysics, 1970. 8, 321-326.

Wojnaroski, L., Bachman, K., \& Pollack, I. High-speed point plotting with computer-controlled displays. Behavioral Research Methods \& Instrumentation, 1971. 3. 210-211.

(Received for publication July 5, 1972:

first revision received October 25, 1972:

second revision received December 27, 1972.) 